\title{
Parameters Identification of Short Hanger Based on Adaptive Genetic Algorithm Pei YUAN ${ }^{1}$
}

${ }^{1}$ China Merchants Chongqing Communications Research \& Design Institute Co., Ltd. Chongqing, China

Keywords: Tension, Short hanger, Genetic Algorithm, Civil engineering.

\begin{abstract}
The hanger tension is crucial in arch bridges, and the fundamental frequency of short hangers is significantly affected by the flexural rigidity. In order to measure the hanger tension considering the flexural rigidity, the Additional Mass Method (AMM) is applied. The tension and flexural rigidity can be calculated by the measured frequencies without and with the additional mass. The difficult inverse calculation among the frequency, tension and stiffness is solved by GA. Using this method to identify the hanger tension and flexural rigidity simultaneous is proved to be feasible through the numerical simulation and field measurement, it is a successful application in civil engineering for computational biology.
\end{abstract}

\section{Introduction}

During the health monitoring and periodical inspection of arch bridges, the tension of hangers is one of the critical parameters to evaluate the structural performance. The common methods of hanger tension measurement mainly include jacking-up method, pressure transducer method, vibration frequency method, and magnetic flux method. Among them, Because of its simplicity and cost-effectiveness, the frequency based method is mostly used in engineering practice. But for short hangers, their natural frequencies are affected not only by tension but also by flexural rigidity and boundary condition, whose effects cannot be ignored. Therefore, it is of great significance to improve the accuracy of vibration frequency in engineering.

To obtain accurate short hanger tension, many diverse solutions [1-4] considering the influence of the flexural rigidity have been proposed as yet. In this paper, the additional mass method [5] based on Genetic Algorithms (GA) is proposed, and validate the methodology by the numerical analysis and the field test of an arch bridge under construction.

\section{The Additional Mass Method (AMM)}

In order to identify the flexural rigidity and the hanger tension simultaneously, the additional mass method (AMM) has been adopted in this paper. Fig. 1 sketches the principle identifying hanger tension using AMM. By a known mass added to the hanger and the location of the added mass, we may obtain some frequencies corresponding to different mass locations. Then the hanger tension $T$ and stiffness $E I$ can be identified by GA. 


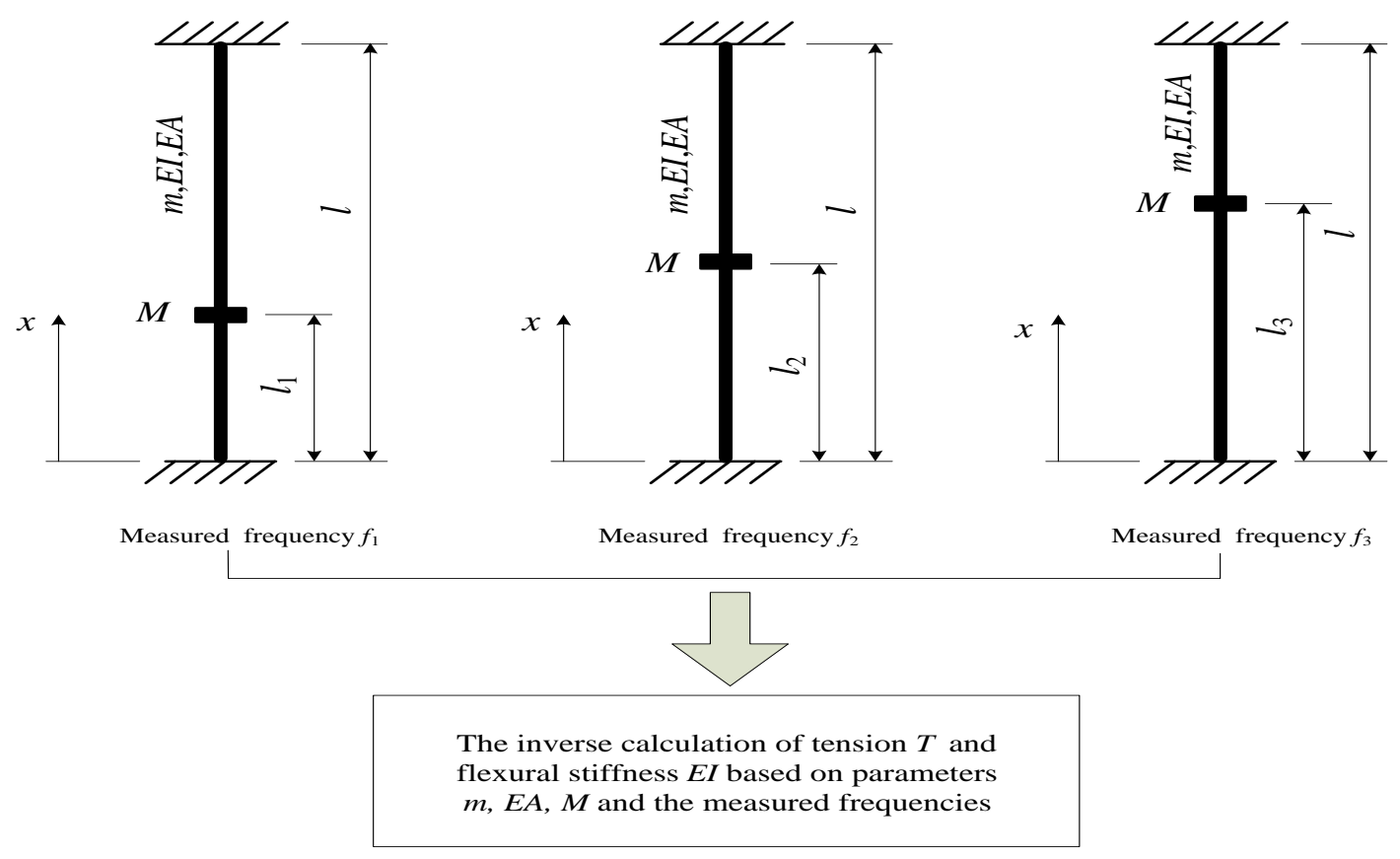

Figure 1. The principle of the additional mass method

\section{Identification and Verification of the Tension Based on GA}

\section{Identification of the Hanger Tension and the Flexural Rigidity}

The key point to identify the hanger tension and the flexural rigidity is calculating the structural parameters according to measured natural frequencies. But the problem is that the inverse calculation is much too difficult. For the problem, this paper attempts Genetic Algorithms which is based on principles of biological evolution. It takes groups of random samples as seeds, and makes samples with higher fitness cross-generate samples to replace samples of lower fitness. According to the biological evolutionism, the new samples generated from the quality samples are more possible to get high quality. And we will get the relatively accurate solution eventually by constantly repeating such a calculating process. The main steps demonstrated as following:

Input the Measured Natural Frequency and Other already Known Parameters. Input the measured hanger natural frequency and other already known parameters. This paper assumes that mass per unit length $(m)$, cross-sectional area $(A)$, elastic modulus $(E)$, length $(l)$, and additional mass position $\left(l_{i}\right)$ is already known. To avoid the influence of measuring error, this paper develops a program which can input multi-group measured parameters. And to get the flexural rigidity and tension, the input data should not be less than 2 groups.

Encoding. Develop the initial sample groups within the set range, and respectively calculate the natural frequency of each sample. Although binary system is commonly used in GA, it is not applicable in hanger parameters. Therefore, this paper adopts real number to denote the chromosome with tension $\mathrm{T}$ and flexural rigidity EI included.

Besides, as the flexural rigidity and tension are of wide value range, it needs a large amount of samples while calculating in the field of positive real numbers. In order to 
avoid the tedious calculation, this paper adopts relative quantity to narrow down the range of the chromosome, it is shown as Eq.1:

$$
E I=s_{1} E I_{o}, T=s_{2} T_{s}
$$

where, $T_{s}$ is the approximate tension on the basis of string vibration theory; $s_{1}$ and $s_{2}$ are the parameters of rigidity and tension respectively. By experience, $s_{1}$ ranges from 0.3 to 1.1 and $s_{2}$ from 0.3 to 1.0. Of course, the ranges of the above parameters are relevant to the specific conditions of hangers. In general, the data above can satisfy the engineering requirements.

Error and Applicability Calculation. According to the frequency by eigenvalue calculation, errors can be denoted as Eq.2.

$$
R_{i}=\sum_{j=1}^{r}\left|\frac{f_{i, j}-f_{j}}{f_{j}}\right|
$$

where, subscript $i$ indicates the chromosome; $f_{i, j}$ indicates the $j$ th natural frequency of group $i ; f_{j}$ is the measured natural frequency; $j$ refers the case, i.e. the measured condition according to the position of the additional mass. We define the fitness of a chromosome as Eq.3:

$$
\text { Fit }_{i}=\frac{1 / R_{i}}{\sum_{j=1}^{n} 1 / R_{j}}
$$

where $\mathrm{Fit}_{i}$ refers to the fitness of group $i$, and $n$ means the total number chromosomes. If the error of the highest fitness satisfies the accuracy demands, the calculation is over astringently.

Crossover and Selection. The chromosomes with poor quality and lower fitness are eliminated according to the pre-set probability. And the genes in the remaining chromosomes are randomly selected to be combined together to obtain the new chromosomes (i.e. perform crossover). In order to maintain the total population, the population of offspring should be equal to the eliminated ones. Besides, to avoid inbreeding, the chromosome of lower fitness among the similar chromosomes should be eliminated before crossing.

Mutation. In order to prevent local convergence and to keep the variety of chromosomes, this paper randomly eliminates some chromosomes every five calculation cycles in a specific proportion, and accordingly generates new chromosomes for complements. What is more, to improve the convergence, the chromosomes with the highest fitness would never be eliminated.

\section{Verification by Numerical Simulation}

Table 1 demonstrates the parameters of the 5 hangers ranging from $3 \mathrm{~m}$ to $10 \mathrm{~m}$. The section of the hangers consists of 91 wires, each with the diameter of $7 \mathrm{~mm}$. The flexural rigidity is assumed to be $200 \mathrm{kN} \cdot \mathrm{m}^{2}$, and the tension is assumed to be $1000 \mathrm{kN}$. In the table, the last two lines show the frequencies in different conditions calculated based on 
finite elements theory. The last but one means the theoretical value of fundamental frequency of hangers themselves, and the last represents the theoretical frequency of hangers with additional mass. In this paper, the boundary constraint assumed to be fixed at both ends.

Table 1. Parameters of hangers used in numerical stimulation

\begin{tabular}{|l|c|c|c|c|c|}
\hline Number & 1 & 2 & 3 & 4 & 5 \\
\hline Length $[\mathrm{m}]$ & 3 & 4 & 6 & 8 & 10 \\
\hline Tension[kN] & 1000 & 1000 & 1000 & 1000 & 1000 \\
\hline Flexural rigidity[kN.m $\left.{ }^{2}\right]$ & 200 & 200 & 200 & 200 & 200 \\
\hline Frequency $[\mathrm{Hz}]$ & 47.79 & 31.90 & 19.02 & 13.55 & 10.53 \\
\hline Frequency with additional mass $[\mathrm{Hz}]$ & 44.31 & 29.27 & 18.48 & 12.51 & 10.41 \\
\hline
\end{tabular}

The experiment is under the condition that the sample scale is 100 groups, the sudden mutation rate is $20 \%$, and the convergence accuracy is $5.0 \times 10^{-5}$. The result is shown in Table 2. In the table, the first line is the tension identified by the method mentioned in this paper, the second line is the flexural rigidity. And the rest is the relative error. The result suggests that the flexural rigidity and the tension of the hanger can be calculated with the accuracy approaching to $100 \%$. In the real case, the boundary of hanger can be considered as fixed. Therefore, the method mentioned in this paper is a valid method in measuring the tension and the flexural rigidity of short hangers.

Table 2. The result of numerical stimulation

\begin{tabular}{|l|c|c|c|c|c|}
\hline Number & 1 & 2 & 3 & 4 & 5 \\
\hline Tension [kN] & 1000.3 & 999.1 & 1001.0 & 1000.2 & 1000.4 \\
\hline Flexural rigidity [kN.m $\left.{ }^{2}\right]$ & 199.9 & 200.4 & 199.3 & 199.7 & 199.4 \\
\hline The error of tension [\%] & 0.0 & 0.1 & 0.1 & 0.0 & 0.0 \\
\hline The error of flexural rigidity [\%] & 0.0 & 0.2 & 0.3 & 0.1 & 0.3 \\
\hline
\end{tabular}

\section{Verification by Field Measurement}

In order to verify the applicability of the method discussed in this paper, a concrete-filled steel tubular arch bridge under construction is taken as a validation example. The tension of two short hangers is calculated by the measured frequency, which is compared with the figure shown on the hydraulic jack.

The upper end of the hanger is fixed at the arch rib, and the lower one at the pre-stressed concrete beam. The section of the arch rib consist of round steel tube of $\Phi \times t=1200 \times 14 \mathrm{~mm}$. The bottom beam is about 1.9 meters high. And both ends have ZM chill casting-anchor head. Table 3 shows the hanger parameters. The flexural modulus is $2.05 \times 10^{5} \mathrm{MPa}$; 7-91 means that the hanger consists of 91 wires with the diameter of 7 $\mathrm{mm}$. Adopting two ring-shaped mass blocks, each single block weighs $10 \mathrm{~kg}$. As is shown in Table 3, frequency is the measured value of fundamental frequency of hangers themselves, and the frequency below is the measured frequency of hangers with additional mass. 
Table 3. The parameters and result of field measurement

\begin{tabular}{|l|c|c|}
\hline number & 1 & 2 \\
\hline Length[m] & 6.175 & 9.120 \\
\hline Type & $7-91$ & $7-55$ \\
\hline Frequency [Hz] & 20.265 & 12.668 \\
\hline Frequency with additional mass [Hz] & 18.268 & 11.471 \\
\hline Height of the additional mass[m] & 3.0 & 3.5 \\
\hline Tension on the jack [kN] & 1600 & 900 \\
\hline Tension identified by GA[kN] & 1576 & 854 \\
\hline Error [\%] & 1.5 & 5.1 \\
\hline
\end{tabular}

This paper adopts the method proposed above to identify the tension. The sample scale is assumed to be 100 groups and the sudden mutation rate 20\%. Table 3 shows the identification result and the comparison of errors. It clearly shows that the identification tension is quite close to the experimental tension, with the error within $6 \%$, which is much better than the string vibration theory.

\section{Conclusion}

This paper shows a kind of vibration method to identify the tension and flexural rigidity of the short hanger. In order to supplement the identification condition, the frequencies with and without additional mass have both been measured. Besides, Genetic Algorithms has been adopted to solve the difficult inverse calculation. This method is proved to be feasible through the numerical simulation and field measurement.

\section{Reference}

[1] Cheng Su, Yufeng Xu and Dajian Han. 2005. Parameter analysis and identification of bending stiffness of cable during tension measurements by frequency method, Journal of Highway and Transportation Research and Development, 22(5): 75-78.

[2] Weifeng Wang and Dajian Han. 2001. The cable tension measurement and parameter identification for cable-stayed bridge, Journal of South China University of Technology (Natural Science Edition), 29(1):18-21.

[3] Wenqing Wu, Chengshu Wang, Guochang Liu et al..2007. Flexible hanger rods tension measurement and parameter identification in large-span tied arch bridge, Journal of Highway and Transportation Research and Development, 24(1): 69-73.

[4] Gang Zheng, Yiqing Ni, Janming Ko et al.. 2005. Theory and implementation of tension tensing and parameter estimation of stay-cables, China civil engineering journal, 38(3): 64-69.

[5] Xu Xie, Liangfeng Sun and Haiyan Huang. 2009. Identification of flexural rigidity and tension of short hangers with adding mass and neural network. ICNC, V3,372-376. 